\title{
Precise Measurements of Hydrogen and Helium Isotopes with BESS-Polar II
}

\author{
N. Picot-Clémente ${ }^{* 11}$, K. Abe ${ }^{2}$, H. Fuke ${ }^{3}$, S. Haino ${ }^{4}$, T. Hams ${ }^{5}$, M. Hasegawa ${ }^{4}$, A. \\ Horikoshi $^{4}$, A. Itazaki ${ }^{2}$, K.C. Kim ${ }^{1}$, T. Kumazawa ${ }^{4}$, A. Kusumoto ${ }^{2}$, M.H. Lee ${ }^{1}$, Y. \\ Makida $^{4}$, S. Matsuda ${ }^{4}$, Y. Matsukawa ${ }^{2}$, K. Matsumoto ${ }^{4}$, J.W. Mitchell ${ }^{5}$, A.A. Moiseev ${ }^{5}$, \\ J. Nishimura ${ }^{1}$, M. Nozaki ${ }^{4}$, R. Orito ${ }^{2}$, J.F. Ormes ${ }^{6}$, K. Sakai ${ }^{5}$, M. Sasaki ${ }^{5}$, E.S. Seo ${ }^{1}$, Y. \\ Shikaze $^{2}$, R. Shinoda ${ }^{7}$, R.E. Streitmatter ${ }^{5}$, J. Suzuki ${ }^{4}$, Y. Takasugi ${ }^{2}$, K. Takeuchi ${ }^{2}$, K. \\ Tanaka $^{4}$, N. Thakur ${ }^{6}$, T. Yamagami ${ }^{4}$, A. Yamamoto ${ }^{4}$, T. Yoshida ${ }^{3}$, K. Yoshimura ${ }^{4}$ \\ 1 Institute for Physical Science and Technology, University of Maryland, College Park MD 20742, \\ USA \\ 2 Kobe University, Kobe, Hyogo 657-8501, Japan \\ 3 Institute of Space and Astronautical Science, Japan Aerospace Exploration Agency \\ (ISAS/JAXA), Sagamihara, Kanagawa 252-5210, Japan \\ 4 High Energy Accelerator Research Organization (KEK), Tsukuba, Ibaraki 305-0801, Japan \\ 5 National Aeronautics and Space Administration, Goddard Space Flight Center (NASA/GFSC), \\ Greenbelt, MD 20771, USA \\ 6 University of Denver, Denver, CO 80208, USA \\ 7 The University of Tokyo, Bunkyo, Tokyo 113-0033, Japan
}

\begin{abstract}
A precise knowledge of cosmic-ray hydrogen and helium isotopes provides important information to better understand Galactic cosmic-ray propagation. Deuteron and helium 3 species are mainly secondary particles created by the spallation of primary proton and helium 4 particles during their propagation in the Galaxy. Secondary-to- primary ratios thus bring direct information on the average amount of material traversed by cosmic rays in the interstellar medium. The Balloonborne Experiment with Superconducting Spectrometer BESS-Polar II flew over Antarctica for 24.5 days from December 2007 through January 2008, during the 23rd solar cycle minimum. The instrument is made of complementary particle detectors which allow to precisely measure the charge, velocity and rigidity of incident cosmic rays. It can accurately separate and precisely measure cosmic-ray hydrogen and helium isotopes between 0.2 and $1.5 \mathrm{GeV} /$ nucleon. These data, which are the most precise to date, will be reported and their implications will be discussed.
\end{abstract}

35th International Cosmic Ray Conference - ICRC2017-

10-20 July, 2017

Bexco, Busan, Korea

\footnotetext{
*Speaker.

${ }^{\dagger}$ Email: picot@umd.edu
} 


\section{Introduction}

The BESS-Polar mission over Antarctica is the successor of the BESS program that carried out 9 northern latitude balloon flights of $\sim 1$ day each from 1993 to 2002 [四]. The BESS-Polar II instrument, presented in this paper, flew over Antarctica between 22 December 2007 and 21 January 2008 during a solar minimum. It recorded 24.5 days of data, containing 4.7 billion particles, more than all previous BESS cumulated flights.

The BESS-Polar experiment is primarily designed to measure low energy antiprotons down to $\sim 100 \mathrm{MeV}[[]$, and search for antinuclei in the cosmic rays [B]]. The instrument is also capable of precisely measuring cosmic-ray fluxes of proton and helium up to $\sim 240 \mathrm{GV}$ [䧃], its Maximum Detectable Rigidity (MDR) ${ }^{1}$. Furthermore, the high velocity and rigidity measurements of the BESS-Polar instrument allow to separate and precisely identify hydrogen and helium isotopes

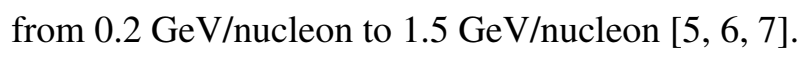

Cosmic-ray ${ }^{2} \mathrm{H}$ and ${ }^{3} \mathrm{He}$ are mainly secondary particles produced through fusion of primary cosmic-ray hydrogen ${ }^{1} \mathrm{H}$ (for ${ }^{2} \mathrm{H}$ ) and fragmentation of ${ }^{4} \mathrm{He}$ (for ${ }^{2} \mathrm{H}$ and ${ }^{3} \mathrm{He}$ ), during their propagation in the interstellar medium. $\mathrm{H}$ and $\mathrm{He}$ isotope fluxes and secondary-to-primary ratio give then crucial information on the cosmic-ray propagation history, with direct information on the amount of interstellar matter traversed in the Galaxy. Such measurements were shown to be as constraining on propagation models as the widely studied secondary-to-primary Boron-to-Carbon ratio [8]. In parallel, they can also bring important information to probe the universality of cosmic-ray propagation for light and heavy elements.

The BESS-Polar instrument will be presented in Section $\S$ ■. The analysis and secondary-toprimary ratios of hydrogen and helium isotope results will be discussed in Section $\S$ 国 and $\S$ 田.

\section{The BESS-Polar Instrument}

The instrument, presented in Figure $\mathbb{W}$, is made of a solenoidal superconducting magnet operated at 0.8 Tesla concentrically arranged with various particle detectors. From top to bottom, a particle traversing the instrument will cross a top time-of-flight hodoscope (TOF), made of plastic scintillators with photomultipliers (PMT) at both ends, a drift-type tracking system made of 4 layers of inner drift chambers (IDC) and a central JET-type drift chamber, a middle TOF counter, an aerogel Cherenkov counter used to separate antiprotons from leptons ${ }^{2}$, and a bottom TOF counter.

Each of the detectors brings information that can fully characterize incident particles. The velocity $\beta$ of the crossing particle is obtained from the time differences between top and bottom (or top and middle) TOFs, with a resolution on $\beta^{-1}$ of $2.5 \%$ for singly-charged particles. The charge $Z$ is measured from ionization energy losses $d E / d x$ recorded in the TOF paddles. The magnetic rigidity $R$ is derived from precise tracking reconstruction of the charged particles deviated in the magnetic field. A resolution of about $0.4 \%$ at $1 \mathrm{GV}$ and a MDR of $240 \mathrm{GV}$ are achieved with the BESS-Polar tracking system. For more detailed information about the BESS-Polar detector, see [Q⿻, [0, ].

\footnotetext{
${ }^{1}$ Beyond the Maximum Detectable Rigidity, curved particle paths can't be distinguished from straight lines.

${ }^{2}$ It rejects $e^{-}$and $\mu^{-}$with a factor of 12000 to identify antiprotons up to $3.5 \mathrm{GeV}$.
} 


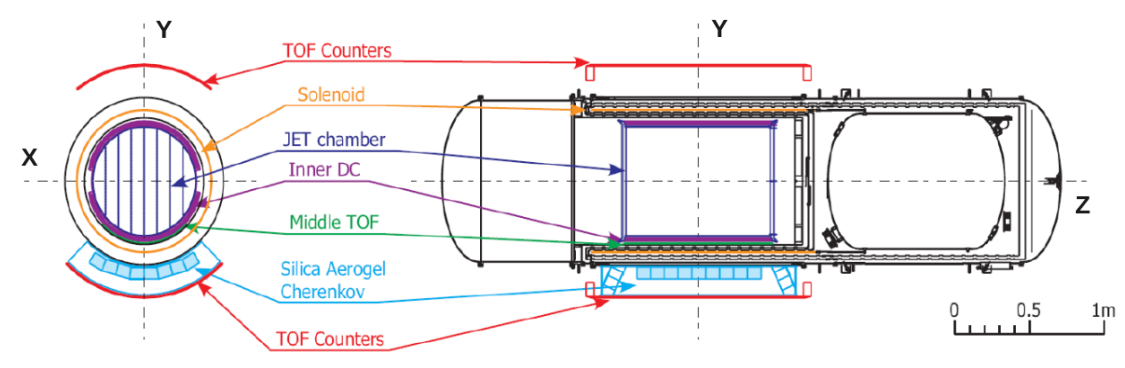

Figure 1: Cross-sectional views of the BESS-Polar II payload.

As a result, after measuring $\beta, Z$, and $R$, the mass $M$ of the crossing particle can be obtained with:

$$
M=Z R \sqrt{1 / \beta^{2}-1} .
$$

The kinetic energy of the particle is obtained with:

$$
E=\sqrt{R^{2} Z^{2}+M^{2}}-M
$$

This kinetic energy is measured inside the instrument, and is therefore then corrected for energy losses in the upper part of the instrument to get the energy at top of instrument. The energy at top of atmosphere is obtained by correcting the energy at top of instrument for ionization energy losses in the atmosphere.

The BESS-Polar II experiment flew at altitudes between $34 \mathrm{~km}$ and $38 \mathrm{~km}$ (average residual atmosphere of $5.8 \mathrm{~g} \mathrm{~cm}^{-2}$ ), at low rigidity cut-off below $0.2 \mathrm{GV}$ and a maximum at $0.6 \mathrm{GV}$. During the stable and long-duration balloon flight of 24.5 days, 2 out of 44 TOF PMTs were turned off due to high voltage issues. High voltage fluctuations that occurred in the central tracker necessitated calibration of the tracker over short periods of time.

\section{Data Analysis}

Precise measurements of $\beta, Z$ and $R$ are needed to separate hydrogen and helium isotopes, thus a rigorous event selection is required. Preselection cuts are defined to keep only downward-going events $(\beta>0$ in top and bottom TOF), and positively charged $(R>0)$ with charge $\mathrm{Z}=1$ or $\mathrm{Z}=2$ in the top TOF. For best TOF measurements, we exclude two paddles (out of 22) that contain only one functioning PMT. From the preselection criteria, we can define here the geometric acceptance of $0.29 \mathrm{~m}^{2} \mathrm{sr}$ at $1 \mathrm{GeV} / \mathrm{n}$, using GEANT4 Monte Carlo [U2] simulations.

For best isotopic separation, it is necessary to remove particles passing outside fiducial regions of the detectors, and that interacted hadronically in the instrument. We defined "single-track" (S.T.) selection criteria for this purpose, which keep only particles with less than 2 hits in the top and bottom TOFs, with single reconstructed tracks that pass through the fiducial area of TOFs and center of the JET chamber, and events with same measured charge in the bottom and top TOF. Moreover to improve isotopic identification, we applied "quality" (Q.) selection cuts to remove particles badly reconstructed due to noise or detector limitations. Selection efficiencies are obtained using flight data sub-samples of charge $Z=1$ or $Z=2$ preselected with the JET chamber. At $1 \mathrm{GeV} / \mathrm{n}$, they are of $\sim 50 \%$ for S.T. cuts and $\sim 95 \%$ for Q. cuts. 
Figure $\square$ shows $\beta^{-1}$ versus $R$ for $Z=1$ and $Z=2$ events after applying the event selection cuts. The figure illustrates the high isotopic separation given by the BESS-Polar II instrument, up to $\sim 4 G V$. Although there is a small discrepancy at the lowest rigidities where limitations of the instrument is reached, the good agreement between theoretical lines and flight data demonstrates that the detectors perform reliable measurements.
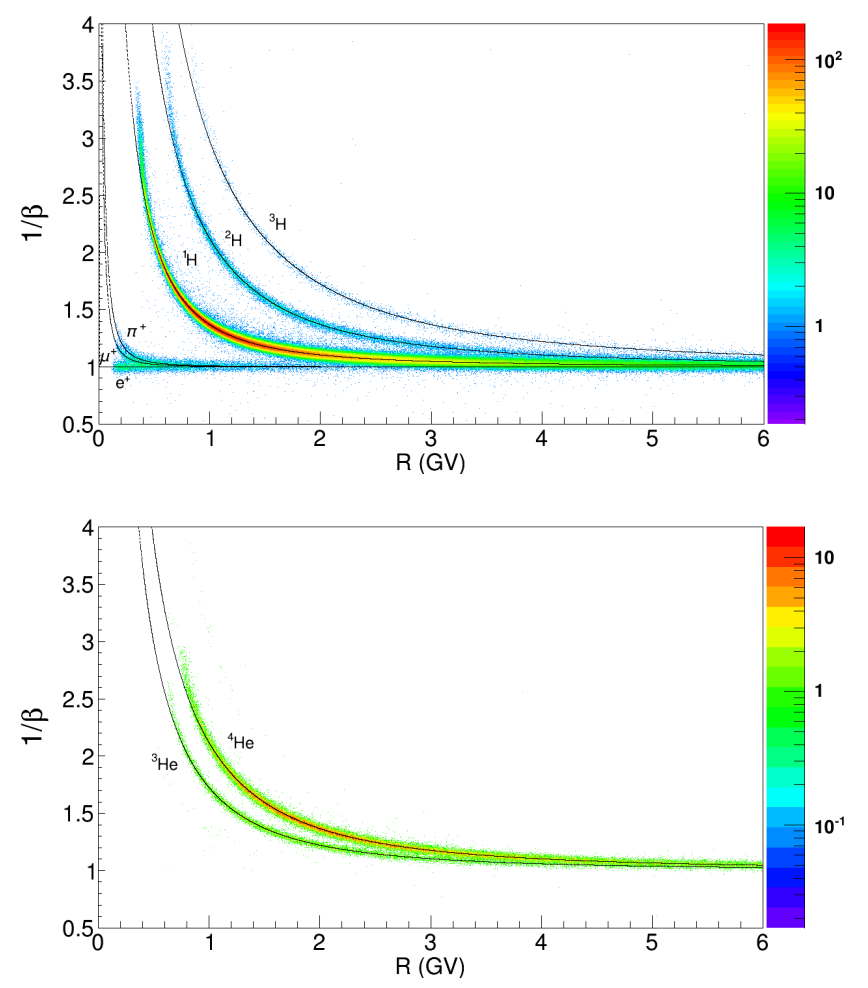

Figure 2: $\beta^{-1}$ versus $R$ for $Z=1$ (top panel) and $Z=2$ (bottom panel) particles after applying the event selection cuts, for $45 \mathrm{~min}$ of data taking. Dashed lines correspond to the theoretical curves for various species present in the data.

Particle's mass distributions obtained from measurements of rigidity $R$, velocity $\beta$ and charge $Z$ are used to identify and count each isotope species. They become more difficult to distinguish with increasing energy and we use double Crystal Ball functions ${ }^{3}$ to fit mass histograms at the highest energies. We can separate and count hydrogen and helium isotopes up to $\sim 1.5 \mathrm{GeV} /$ nucleon.

\section{Results}

Secondary-to-primary ratios of ${ }^{2} \mathrm{H} /{ }^{1} \mathrm{H}$ and ${ }^{3} \mathrm{He} /{ }^{4} \mathrm{He}$ can give important information on cosmicray propagation in the Galaxy. For same charge species, ratio measurements are quasi-independent to corrections of geometric acceptance, efficiencies and atmospheric secondaries. Figure $B$ and $\mathbb{G}$ show secondary-to-primary ratios of ${ }^{2} \mathrm{H} /{ }^{1} \mathrm{H}$ and ${ }^{3} \mathrm{He} /{ }^{4} \mathrm{He}$ measured by BESS-Polar II in the energy range from 0.2 to $1.5 \mathrm{GeV} / \mathrm{n}$, and compared to previous results from IMAX-92 [[13], BESS-93 [[]],

\footnotetext{
${ }^{3}$ A Crystal Ball function consist of a Gaussian function with a power-law tail.
} 
AMS-01 [14]], BESS-00 [目] and PAMELA [15]]. For comparison, the GALPROP code [ए6], in which the proton fusion process was implemented and isotope production cross sections were improved in the energy range of interest (c.f. [ए]]), was computed using stochastic reacceleration [[1]] at different solar modulation parameters of 450, 700, $1200 \mathrm{MV}$ (force-field model).

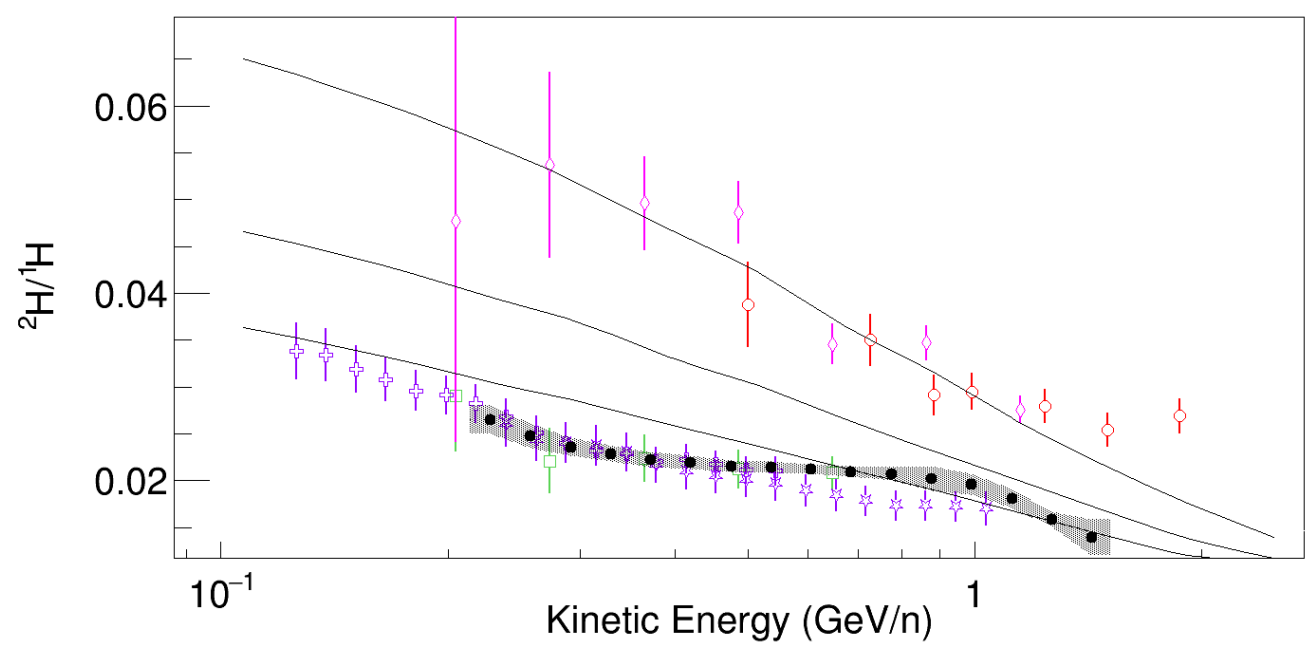

Figure 3: Measured ratio of ${ }^{2} \mathrm{H} /{ }^{1} \mathrm{H}$ with BESS-Polar II (black filled circles). Measurements are compared with IMAX-92 [[13] (open circles), BESS-93 [可] (open squares), BESS-00 [6] (open diamonds) and PAMELA [45] (open stars and crosses). GALPROP [ए6], in which the proton fusion process was implemented and isotope production cross sections were updated (c.f. [[]]), is employed for comparison using stochastic reacceleration [पष]] (solid lines) at different solar modulation parameters of 450, 700 and 1200 MV (force-field model).

BESS-Polar II secondary-to-primary isotope ratios are the most precise in the energy range of $0.2 \mathrm{GeV} / \mathrm{n}$ to $1.5 \mathrm{GeV} / \mathrm{n}$. As expected for same solar activity, both BESS-Polar II and PAMELA ratios of ${ }^{2} \mathrm{H} /{ }^{1} \mathrm{H}$ are consistent. Results follow solar modulation expectations which predict low ratios at solar minimum and high ratios at solar maximum (observed with IMAX-92 and BESS-00). BESS-93 data, although taken at higher solar activity, are consistent within their large uncertainties with BESS-Polar II. Ratios of ${ }^{3} \mathrm{He} /{ }^{4} \mathrm{He}$ are less affected by solar modulations as the A/Z ratio of the two species are similar. Within uncertainties, AMS-01 and BESS-93 are consistent with BESS-Polar II, while PAMELA results exhibit a significant discrepancy, with a much lower ratio than previous measurements. The GALPROP code [16, [7], using stochastic reacceleration, with diffusion following Kolmogorov turbulence, was used for predictions. Expectations fits generally well BESS-Polar II measurements with same solar modulation of $450 \mathrm{GV}$.

The new precise $\mathrm{H}$ and $\mathrm{He}$ isotope measurements from BESS-Polar II bring important information to better understand cosmic-ray propagation. Predictions using GALPROP generally match well measurements but leave room for further improvements to better reproduce observations, which bring new significant constraints on propagation parameters and models.

\section{Conclusion}

The BESS-Polar II experiment offers unique data to significantly improve our knowledge on 


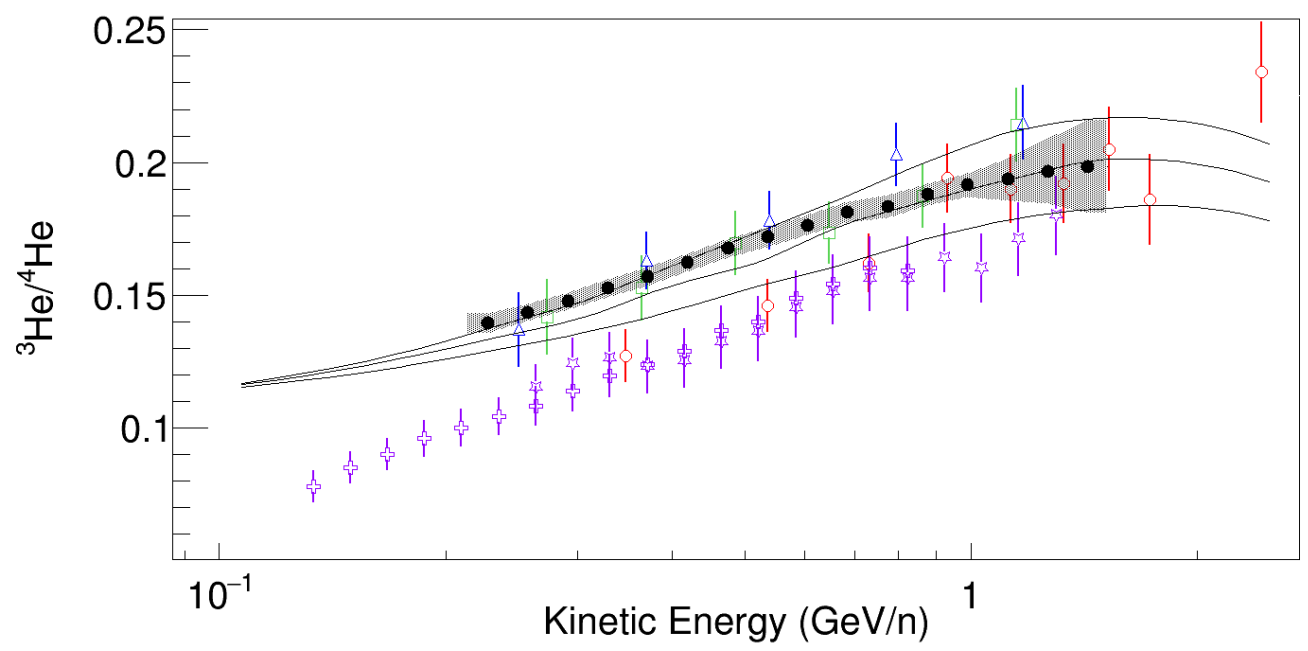

Figure 4: Measured ratio of ${ }^{3} \mathrm{He} /{ }^{4} \mathrm{He}$ with BESS-Polar II (filled circles). Measurements are compared

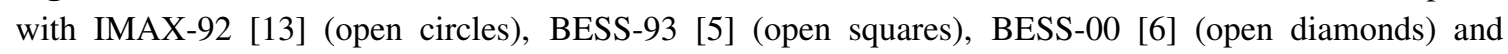
PAMELA [15] (open stars and crosses). GALPROP [16], in which the proton fusion process was implemented and isotope production cross sections were updated (c.f. [[D]]), is employed for comparison using stochastic reacceleration [1]8] (solid lines) at different solar modulation parameters of 450, 700 and 1200 MV (force-field model).

cosmic-ray propagation in the interstellar medium. In this paper were presented cosmic-ray hydrogen and helium isotope measurements of secondary-to-primary ratios with BESS-Polar II. Results are the most precise to date between $0.2 \mathrm{GeV} /$ nucleon and $1.5 \mathrm{GeV} /$ nucleon, and show a good consistency with previous measurements and solar modulations, except with PAMELA ${ }^{3} \mathrm{He} /{ }^{4} \mathrm{He}$. For comparison, predictions including stochastic reacceleration were computed using a modified version of GALPROP more suitable for studying the light-quartet isotopes. The reacceleration model used in this paper fits generally well both ${ }^{2} \mathrm{H} /{ }^{1} \mathrm{H}$ and ${ }^{3} \mathrm{He} /{ }^{4} \mathrm{He}$ measurements. However, the high precision isotope measurements from BESS-Polar II show that there is still room for further model improvements. These results bring new opportunities to better constrain cosmic-ray propagation models and parameters.

\section{Acknowledgements}

This work was supported by NASA grant NNX15AC16G and its predecessors. The BESSPolar experiment is a U.S.-Japan collaboration supported by NASA in the US and KAKEN-Hi in Japan. The authors thank NASA Headquarters, the NASA Balloon Program Office at Goddard Space Flight Center/Wallops Flight Facility, and the NASA Columbia Scientific Balloon Facility, as well as ISAS/JAXA and KEK for their support. Thanks to the US National Science Foundation (NSF) and Raytheon Polar Services Company for their professional support in the USA and in Antarctica. Thanks to the GALPROP team, supported through NASA grants and by the Max Planck Society, for providing the program to the scientific community (http://galprop.stanford.edu/). 


\section{References}

[1] A. Yamamoto et al., Search for cosmic-ray antiproton origins and for cosmological antimatter with BESS, Adv. Space Res. 51 (2), 227, 2013.

[2] K. Abe et al., Measurement of the cosmic-ray antiproton spectrum at solar minimum with a long-duration balloon flight over Antarctica, Phys. Rev. Lett. 108, 051102, 2012.

[3] K. Abe et al., Search for antihelium with the BESSpolar Spectrometer, Phys. Rev. Lett. 108, 131301, 2012.

[4] K. Abe et al., Measurements of cosmic-ray proton and helium spectra from the BESS-Polar long-duration balloon fights over Antarctica, Astrophys. J. 822 (2), 65, 2016.

[5] J. Z. Wang et al., Measurement of cosmicray hydrogen, helium, and their isotopic composition with the BESS experiment, Astrophys. J. 564, 244, 2002.

[6] K.C. Kim et al., Cosmic ray 2H/1H ratio measured from BESS in 2000 during solar maximum, Adv. Space Res. 51 (2), 234, 2013.

[7] K. Abe et al., Time variations of cosmic-ray helium isotopes with BESS-Polar I, Adv. Space Res., Adv. Space Res. 53 (10), 1426, 2014.

[8] B. Coste, L. Derome, D. Maurin and A. Putze, Constraining Galactic cosmic-ray parameters with Z $\leq 2$ nuclei, A\&A 539, A88, 2012.

[9] S. Haino et al., Progress of the BESS Superconducting Spectrometer, Nucl. Instrum. Methods A518, 167, 2004.

[10] T. Yoshida et al., BESS-polar experiment, Adv. Space Res. 33 (10), 75, 2004.

[11] K. Abe, H. Fuke, S. Haino, et al. Measurement of the cosmic-ray low-energy antiproton spectrum with the first BESS-polar Antarctic flight, Phys. Lett. B670, 103, 2008.

[12] Application Software Group, GEANT, Detector description and simulation tool, CERN program library long writeup W5013, 1993. http://wwwasd.web.cern.ch/wwwasd/geant/

[13] W. Menn et al., The Absolute Flux of Protons and Helium at the Top of the Atmosphere Using IMAX, Astrophys. J. 533, 281, 2000.

G.A. De Nolfo et al., A measurement of cosmic ray deuterium from 0.5-2.9 GeV/nucleon, AIP Proc. 528, 425, 2000.

[14] M. Aguilar et al., Isotopic Composition of Light Nuclei in Cosmic Rays: Results From AMS-01, Astrophys. J. 736, 105, 2011.

[15] O. Adriani et al., Measurements of Cosmic-Ray Hydrogen and Helium Isotopes with the PAMELA Experiment, Astrophys. J. 818, 1, 2016.

[16] A. W. Strong, I. V. Moskalenko, Propagation of Cosmic-Ray Nucleons in the Galaxy, Astrophys. J. 509, 212, 1998.

[17] N. Picot-Clémente, E. S. Seo, A. Strong, Y. Lu, Study of Cosmic-Ray Transport with the GALPROP Code, Proc. 34rd Int. Cosmic Ray Conf., ID 1267, 2015.

[18] E. S. Seo and V. S. Ptuskin, "Stochastic Reacceleration of Cosmic Rays in the Interstellar Medium", Astrophys. J. 431, 705, 1994. 